\title{
Analyse contextuelle des politiques à l'appui de l'auto-prise en charge des maladies chroniques au Canada
}

\author{
C. Liddy, M.D. (1, 2); K. Mill, B. Sc. (1)
}

Cet article a fait l'objet d'une évaluation par les pairs.

\section{Résumé}

Introduction : Les données probantes appuyant l'auto-prise en charge des maladies chroniques méritent de faire l'objet d'une analyse plus approfondie. Notre objectif était de cerner les politiques, les stratégies et les cadres existants à l'appui des initiatives d'auto-prise en charge.

Méthodologie : La présente étude descriptive a été effectuée sous forme d'analyse contextuelle. Elle comprend une recherche sur les sites Web gouvernementaux et d'autres sites Web accessibles au public ainsi que des entrevues avec des représentants gouvernementaux choisis par l'entremise du Conseil canadien de la santé et par réseautage universitaire.

Résultats : Nous avons interviewé 16 représentants de toutes les provinces et de tous les territoires du Canada et nous avons relevé 30 documents provinciaux et nationaux d'intérêt accessibles au public. La plupart des provinces et des territoires possèdent des politiques dont certains éléments portent sur l'auto-prise en charge des maladies chroniques. Les politiques de l'Alberta et de la Colombie-Britannique sont les plus détaillées. Elles accordent une place de choix aux soins primaires et elles ne sont pas axées sur une maladie en particulier. Elles présentent également des mesures pour la mise en œuvre à l'échelle provinciale des programmes d'auto-prise en charge des maladies chroniques. L'ensemble des territoires du nord du Canada n'avait pas de politiques précises sur l'auto-prise en charge des maladies chroniques, malgré un lourd fardeau associé à ces maladies.

Conclusion : Faire participer les patients à l'auto-prise en charge de leur maladie chronique est important et efficace. Même si la plupart des provinces et des territoires possèdent des politiques qui comportent des éléments liés à l'auto-prise en charge des maladies chroniques, ces politiques sont souvent intégrées à d'autres initiatives ou d'autres documents de politiques axés sur des maladies ou des populations en particulier, ce qui peut limiter la portée et l'effet potentiels de l'auto-prise en charge.

Mots-clés : auto-prise en charge des maladies chroniques, soutien à l'auto-prise en charge, politique en matière de santé, soins primaires, analyse contextuelle

\section{Introduction}

Les maladies chroniques constituent le principal problème lié aux soins de santé au Canada: elles coûtent plus de 80 milliards de dollars chaque année $e^{1,2}$ et entraînent une utilisation accrue des services des urgences, des hospitalisations prolongées, une diminution de la qualité de vie et une augmentation des taux de

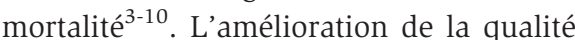
des soins pour les personnes atteintes de maladies chroniques est complexe ${ }^{11}$, car elle nécessite un diagnostic et un traitement rapides, un accès aux soins primaires et aux soins spécialisés ainsi qu'une attention particulière accordée aux tâches et aux décisions liées à l'autoprise en charge $\mathrm{e}^{12,13}$.

Il a été établi que le soutien à l'auto-prise en charge améliore les résultats, aussi cette intervention a-t-elle été préconisée pour un large éventail de maladies et de populations $^{14-20}$. Le soutien à l'auto-prise en charge est axé sur les personnes et leurs familles; il mise sur l'établissement concerté d'objectifs et sur une gamme de stratégies d'auto-efficacité ${ }^{16}$. Ces stratégies permettent au patient, en collaboration avec les fournisseurs de soins de santé, de prendre en charge médicalement leur maladie de manière plus efficace, d'exercer leurs activités et leurs rôles habituels, ainsi que de maîtriser les répercussions émotionnelles de leur maladie $^{15}$. Adams et collab. complètent cette définition en soulignant ce qui peut être fait par les fournisseurs de soins de santé par le biais " [traduction] de la mise en œuvre systématique d'activités d'éducation et de soutien par les professionnels de la santé ${ }^{21}$, p. 57 pour améliorer les compétences et la confiance des patients en ce qui concerne la prise en charge de leurs problèmes de santé, notamment l'évaluation régulière des progrès réalisés et des problèmes, la définition d'objectifs et l'aide à la résolution de problèmes.

La mise en œuvre de programmes de soutien à l'auto-prise en charge au Canada suscite beaucoup d'intérêt. Toutefois, de nombreux programmes sont mis en place 
isolément, souvent par des organismes axés sur une maladie en particulier, par les autorités de santé publique locales ou des organismes communautaires ${ }^{22}$. Par contre, bien que les patients et leur communauté, les fournisseurs de soins de santé et le système de santé soient des acteurs clés du succès du soutien et des soins liés aux maladies chroniques, les gouvernements fédéraux, provinciaux et territoriaux ont également un rôle majeur à jouer parce qu'ils élaborent et mettent en œuvre des politiques publiques en matière de santé et de soins de santé à l'échelle du Canada.

Même si l'importance de l'auto-traitement et de l'auto-prise en charge est parfois mentionnée dans des stratégies nationales, par exemple le programme sur le vieillissement en santé ${ }^{23}$ et la Stratégie canadienne du diabète ${ }^{24}$, on en sait peu à propos de l'orientation des politiques provinciales et territoriales en matière de soutien à l'auto-prise en charge, même si la santé et les soins de santé sont du ressort des gouvernements provinciaux et territoriaux.

Dans le cadre d'un projet plus vaste portant sur les soins liés aux maladies chroniques et l'auto-prise en charge réalisé avec le Conseil canadien de la santé $(\mathrm{CCS})^{25}$, nous avons effectué une analyse contextuelle en vue de relever les documents de politiques stratégiques provinciales et territoriales appuyant l'auto-prise en charge par le patient ${ }^{26}$. Le CCS est un organisme à but non lucratif et indépendant mis en place par les premiers ministres du pays en 2003 et chargé de surveiller le système de santé dans le cadre des Accords sur la santé. Le CCS s'est entre autres penché sur la prévention et la prise en charge des affections chroniques en vue d'encourager la discussion sur les modifications des politiques publiques, la gestion des soins de santé et la prestation des services de santé en vue d'améliorer les résultats liés à la santé pour l'ensemble des Canadiens ${ }^{27}$.

Le présent rapport vise à améliorer la connaissance des activités et des orientations stratégiques provinciales afin de permettre aux provinces et aux territoires de s’inspirer des tendances émergentes à l'échelle du pays.

\section{Méthodologie}

L'analyse contextuelle du soutien à l'autoprise en charge et des soins liés aux maladie chronique s'est déroulée en trois phases: 1) une analyse en ligne à l'aide du moteur de recherche Google pour repérer les politiques accessibles au public qui appuient ou influencent les initiatives de soutien à l'auto-prise en charge; 2) des entrevues avec des représentants provinciaux ou territoriaux du CCS pour obtenir un point de vue interne au sujet des politiques et des stratégies existantes ainsi que de la planification à venir touchant le soutien à l'auto-prise en charge et 3) une seconde analyse des documents en ligne en fonction des résultats des entrevues.

La première analyse en ligne visait à repérer les documents de politiques provinciales et territoriales accessibles au public. Le terme " politique » a été défini comme suit: tout plan d'action ou document d’orientation général appuyé par une autorité gouvernementale, notamment les cadres, les stratégies, les plans d'action et les documents de priorité officiels ${ }^{28}$.

En septembre 2011, trois personnes de notre équipe de recherche ont parcouru les documents en ligne et les sites Web de chaque province et territoire en vue de repérer les politiques, les lois, les stratégies et les cadres qui concernaient, ou mentionnaient, le soutien à l'auto-prise en charge, les programmes connexes ou leur mise en œuvre. Les mots-clés de recherche utilisés étaient : " self-management ", " self-care ", " self-management support ", " chronic conditions ", " policy ", " action plan », " framework ", "strategy » et " initiative ». Les résultats pertinents ont été compilés dans une base de données à l'aide de la version 12 du logiciel Microsoft Excel (2007; Redmond, Washington, États-Unis), avec l'indication de l'année de chaque initiative et des détails la concernant.

Par la suite, pour obtenir un portrait plus détaillé et exact des politiques existantes, nous avons interviewé des personnes participant à l'élaboration des politiques au sein des ministères de la Santé. Des représentants de toutes les provinces et de tous les territoires, à l'exception du
Québec, ont été choisis par l'entremise du réseau du CCS et invités par courriel à participer à une entrevue téléphonique de 30 minutes. Comme le Québec ne collaborait alors pas officiellement avec le CCS, le représentant du Québec a été trouvé par réseautage universitaire. Tous les représentants invités ont accepté de participer et ont fourni un consentement éclairé. Le processus d'entrevue a été approuvé par le comité d'éthique de la recherche de l'hôpital d'Ottawa.

Les auteurs peuvent fournir, sur demande, le guide d'entrevue utilisé pour réaliser les entrevues semi-dirigées. La chercheure principale (CL) ou l'assistante de recherche (KM) ont effectué les entrevues entre septembre et octobre 2011, l'entrevue avec le représentant du Québec ayant eu lieu quant à elle en mai 2012. Les entrevues ont été enregistrées et transcrites par l'assistante de recherche. Une copie de la transcription des entrevues a été envoyée à chaque personne interviewée pour obtenir son approbation et améliorer la fiabilité des résultats.

La troisième étape de l'étude, qui s'est déroulée en juillet 2012, a consisté en une analyse en ligne ciblée visant à repérer des documents de politiques récemment publiés ou mis à jour dont la parution avait été annoncée par les représentants interviewés. L'analyse itérative a utilisé des exemples d'autres analyses de politiques à des fins d'orientation ${ }^{29-31}$. Conformément aux travaux de Dixon-Woods et collab. ${ }^{32}$, nous avons utilisé une approche narrative descriptive associée à une analyse thématique. Cette approche a été jugée appropriée à une analyse axée sur les politiques $^{32}$. Deux membres de l'équipe de recherche ont passé en revue les documents de politiques et les transcriptions des entrevues afin d'en cerner les thèmes. Plusieurs rencontres d'équipe ont eu lieu au cours de la phase d'analyse afin de discuter des résultats et de définir les thèmes clés ${ }^{32}$.

\section{Résultats}

Grâce à l'analyse en ligne et aux entrevues avec 16 représentants des provinces et des territoires canadiens, nous avons appris que la plupart des provinces et des 
territoires avaient une politique, un cadre ou une stratégie qui intégrait certains aspects de la prise en charge des maladies chroniques. Toutefois, le nombre de documents de politiques accessibles qui reconnaissaient explicitement le rôle de l'autoprise en charge a varié considérablement d'une province et d'un territoire à l'autre (tableau 1). Notre analyse des documents en ligne visant à repérer les politiques qui appuient ou influencent les initiatives de soutien à l'auto-prise en charge a permis de relever 30 documents provinciaux et nationaux pertinents et accessibles au public.

La majorité des provinces ont mis en œuvre des programmes de soutien à l'auto-prise en charge, le plus courant étant le programme d'auto-prise en charge des maladies chroniques de l'Université de Stanford (tableau 2). Toutefois, ces programmes sont souvent gérés par de petits organismes communautaires ou par les autorités sanitaires locales. L'Alberta et la ColombieBritannique sont les provinces qui possèdent les politiques les plus détaillées à l'appui de l'auto-prise en charge par le patient. Leurs programmes d'auto-prise en charge, largement accessibles, sont principalement offerts par l'entremise d'organismes sanitaires provinciaux (et non par celle de groupes axés sur une maladie en particulier ou de groupes communautaires). Ces programmes mettent l'accent sur les soins centrés sur les patients et englobent les soins de santé primaires et les soins primaires.

Par exemple, l'Alberta s'est dotée d'une vision globale pour l'avenir des soins de santé, appelée Vision 2020, qui est axée sur les besoins des patients ${ }^{33}$. De plus, l'élaboration de son modèle de gestion des soins en cas de maladie chronique et le lancement de programmes communautaires intégrés à l'échelle de la province visent à promouvoir une approche équilibrée du soutien aux patients atteints d'affections chroniques. Le soutien à l'auto-prise en charge est l'un des principaux piliers du modèle et de l'élaboration des programmes. Le programme d'autoprise en charge des maladies chroniques de l'Université de Stanford est maintenant offert à l'échelle de la province par les services de santé de l'Alberta sous le nom de Better Choices, Better Health. Il fait partie des programmes communautaires et est offert aux patients par leur médecin ou par le personnel des autres programmes intégrés. Ces derniers comprennent des programmes supervisés d'activité physique et de l'information sur la nutrition offerts par un nutritionniste ou dans le cadre d'un atelier de groupe. Les réseaux de soins primaires en Alberta encouragent aussi fortement l'auto-prise en charge. Les réseaux jouent un rôle important dans le cadre des programmes communautaires intégrés en raison de leur capacité à améliorer la coordination des soins et la collaboration fondée sur le partage de la prestation des soins entre les fournisseurs de soins appropriés.

De manière similaire, l'auto-prise en charge est mentionnée dans la mission, la vision et les objectifs du ministère de la Santé de la Colombie-Britannique. L'initiative ministérielle, Patients as Partners, qui fait partie de la charte des soins de santé primaires de $2007^{34}$, aborde spécifiquement la mise en œuvre et l'évaluation de l'auto-prise en charge en invitant les fournisseurs de soins de santé primaires et les organismes à élaborer de nouveaux moyens d'appuyer le rôle central des patients à titre de partenaires de leur propre traitement. Cette province offre de nombreux programmes de soutien à l'auto-prise en charge, notamment les suivants : Chronic Disease SelfManagement; Online Chronic Disease Self-Management; Arthritis/Fibromyalgia Self-Management; Chronic Pain SelfManagement; Diabetes Self-Management; Active Choices; A Matter of Balance: Managing Concerns about Falls; Bounce Back: Reclaim Your Health; InterCultural Online Health Network; Patient Voices Network's Peer Coaching; Dietician Services at HealthLink BC et QuitNow Services.

Le Manitoba a aussi publié récemment un document de travail portant spécifiquement sur l'auto-prise en charge dans le cadre des soins primaires.

\section{Cadres}

De nombreuses autres provinces se sont dotées de cadres pour la prise en charge et la prévention des maladies chroniques dans lesquels l'auto-prise en charge est un élément central. Par exemple, l'Ontario, le Nouveau-Brunswick et le Québec ont harmonisé leurs cadres de prise en charge et de prévention des maladies chroniques, qui sont fondés sur le modèle élargi de soins aux malades chroniques Exanded Chronic Care Model $^{34,35}$, afin d'élaborer des stratégies et des politiques futures pour la prévention et la prise en charge des maladies chroniques. Ce modèle élargi s'appuie sur le modèle bien connu de soins aux malades chroniques Chronic Care Model $^{36}$, qui s'est avéré efficace pour améliorer la prestation et la qualité des soins ainsi que le contrôle des coûts des soins de santé ${ }^{14,19,37}$. Le modèle Exanded Chronic Care Model convient mieux à l'environnement des soins de santé canadien, car il intègre plus efficacement la promotion de la santé et la prévention tant à l'intérieur du système de santé que dans les collectivités.

Terre-Neuve-et-Labrador a également adopté un cadre stratégique sur les maladies chroniques, qui contient six énoncés de principes, dont l'un portant sur l'autoprise en charge ${ }^{38}$. Il vise huit priorités: l'arthrite, le cancer, la douleur chronique, le diabète, les cardiopathies, les maladies pulmonaires, les maladies du rein et l'accident vasculaire cérébral (AVC). Il est valable pour les quatre autorités régionales de santé de la province.

\section{Stratégies}

L'Unité de santé des populations et de prévention des maladies chroniques de l'Université Dalhousie, en collaboration avec le ministère de la Santé de la Nouvelle-Écosse, a élaboré la Nova Scotia Chronic Disease Prevention Strategy en 2003, mais cette stratégie ne met toutefois pas explicitement l'accent sur l'auto-prise en charge. La Strategy for Positive Aging in Nova Scotia, publiée en 2005, souligne quant à elle l'importance de l'auto-prise en charge chez les personnes âgées.

\section{Politiques portant sur une maladie en particulier axées sur l'auto-prise en chargé}

De nombreuses provinces possèdent des politiques axées sur des maladies en 


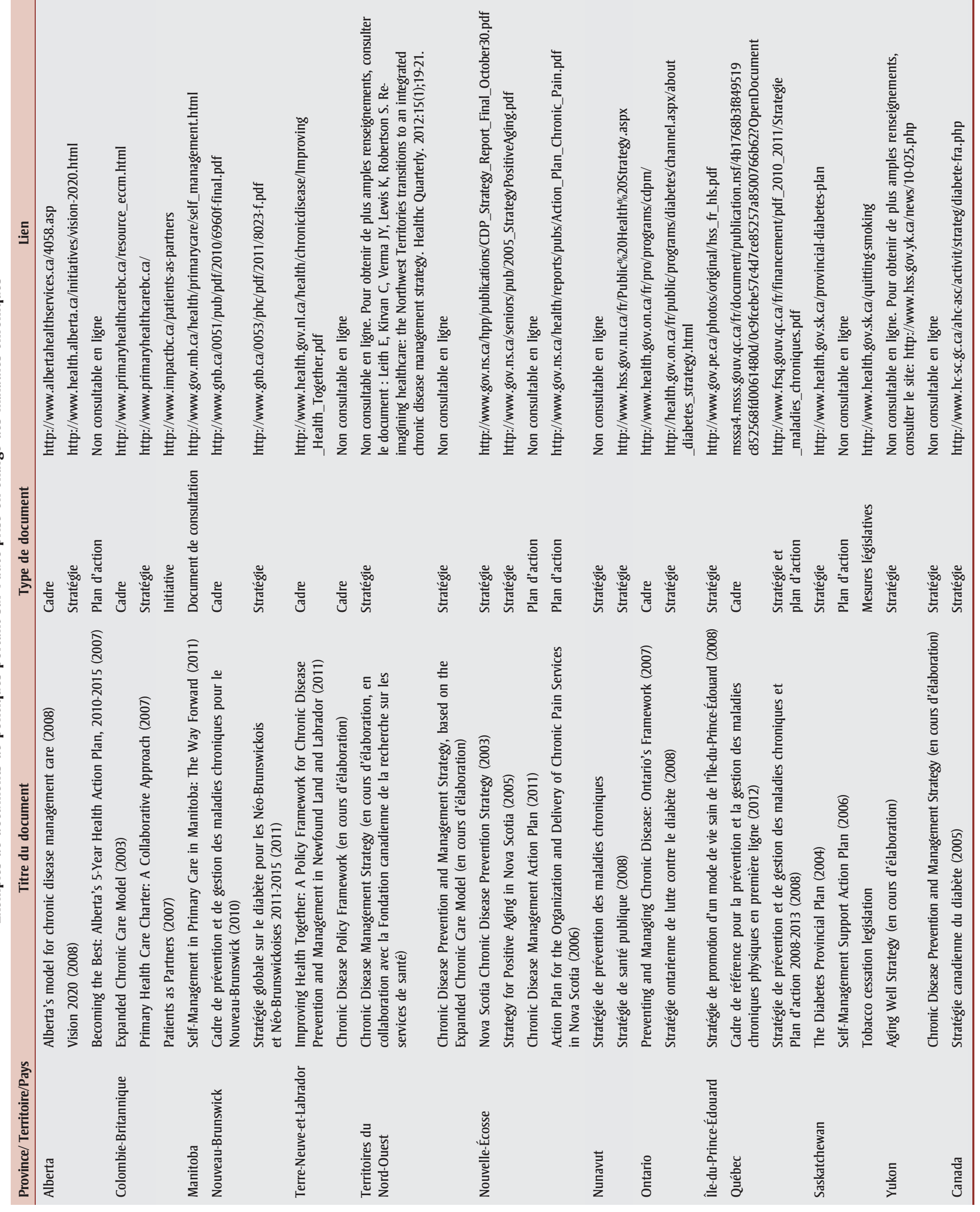




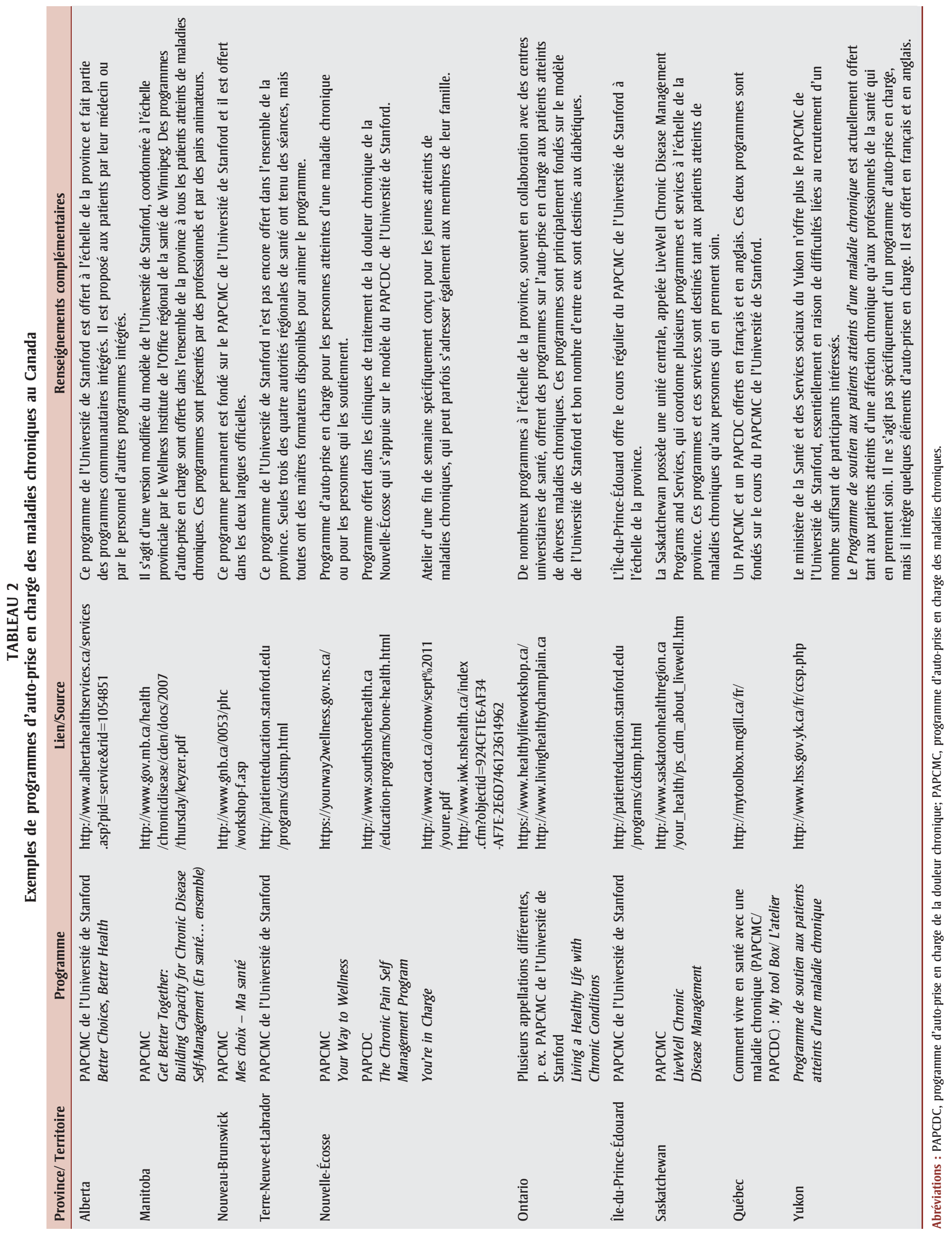


particulier, comme le diabète, l'arthrite, l'AVC ou la maladie pulmonaire obstructive chronique. Par exemple, la Stratégie ontarienne de lutte contre le diabète, lancée en 2008, met l'accent sur l'importance de l'auto-prise en charge. Dans le cadre de cette stratégie, du financement a été accordé à un plan quadriennal afin de mettre en place une approche multidimensionnelle des soins liés au diabète en vue de répondre aux besoins grandissants de la population ontarienne. La Stratégie ontarienne de lutte contre le diabète est la stratégie incluant l'auto-prise en charge la plus importante en Ontario. Toutefois, les experts du domaine interviewés ont souligné qu'ils croient en la nécessité d'aller au-delà d'une stratégie liée à une maladie pour formuler une politique plus globale qui porte sur l'auto-prise en charge des maladies chroniques en général, en particulier chez les patients atteints d'affections comorbides.

Le Provincial Diabetes Plan de la Saskatchewan, publié en février 2004, souligne le rôle de l'auto-prise en charge. Le ministère de la Santé de la Saskatchewan et les autorités locales de santé publique ont également mis en place des lignes directrices qui prescrivent la prestation de soutien à l'auto-prise en charge.

À l'̂̂le-du-Prince-Édouard, l'auto-prise en charge de certaines maladies chroniques, notamment le diabète et l'arthrite, est également ciblée par certains programmes. La province a aussi mis à l'essai des programmes visant la maladie pulmonaire obstructive chronique, l'hypertension et la gestion du poids, qui comportent des volets liés à l'auto-prise en charge. L'Île-du-Prince-Édouard n'a pas de document de politiques précis à l'appui de l'auto-prise en charge des maladies chroniques en général. La province offre plutôt de l'éducation et de la formation aux fournisseurs de soins en ce qui a trait aux principes de l'auto-prise en charge.

\section{Lacunes des politiques, des cadres et des stratégies dans le Nord}

\section{Nunavut}

Notre analyse des politiques, étayée par l'entrevue que nous avons menée auprès de l'expert local du Nunavut, a révélé que le territoire ne possède pas de documents de politiques ni de stratégies qui portent spécifiquement sur l'auto-prise en charge par les patients atteints de maladies chroniques. De plus, il n'y a actuellement aucun programme d'auto-prise en charge actif visant les patients ou les professionnels de la santé au Nunavut.

\section{Territoires du Nord-Ouest}

Aucune politique n'est en place dans les Territoires du Nord-Ouest pour appuyer spécifiquement l'élaboration et la mise en place de programmes d'auto-prise en charge destinés aux patients atteints de maladies chroniques, même si une stratégie de prise en charge des maladies chroniques est actuellement élaborée par le ministère de la Santé et des Services sociaux et qu'une première ébauche du document a été rédigée et est en cours d'examen. Le soutien à l'auto-prise en charge y est reconnu comme un élément important. Un nombre limité de programmes de la région intègrent totalement l'auto-prise en charge: certains programmes d'éducation sur le diabète et un petit nombre d'autres programmes concernant des maladies en particulier, dont des programmes de santé mentale. Une stratégie de prise en charge des maladies chroniques sera l'occasion d'accroître la part de l'auto-prise en charge dans ces programmes et de concevoir de nouveaux programmes qui répondent mieux au besoin en matière de soutien à l'auto-prise en charge dans les Territoires du Nord-Ouest.

\section{Yukon}

Le ministère de la Santé et des Services sociaux a fait une demande de financement afin d'entreprendre l'élaboration d'une stratégie de prévention et de prise en charge des maladies chroniques. Selon les experts interviewés, on se propose d'inclure l'auto-prise en charge dans la stratégie. Le programme d'auto-prise en charge des maladies chroniques de l'Université de Stanford n'est plus offert par le ministère de la Santé et des Services sociaux, principalement en raison des difficultés liées au recrutement d'un nombre suffisant de patients intéressés. Le Programme de soutien aux patients atteints d'une maladie chronique est offert tant aux patients atteints de maladies chroniques qu'aux professionnels de la santé qui en prennent soin. Il ne s'agit pas principalement d'un programme d'autoprise en charge, mais il comprend certains éléments qui y sont reliés.

\section{Analyse}

Dans le cadre de notre analyse contextuelle des politiques, nous avons noté que même si la plupart des provinces et des territoires possèdent des politiques qui comportent des éléments liés à l'autoprise en charge des maladies chroniques, ces politiques étaient souvent intégrées à d'autres initiatives ou documents de politiques conçus pour des populations ou des maladies en particulier. L'absence de politiques axées sur l'auto-prise en charge dans l'ensemble du Nord canadien est surprenante, étant donné que le fardeau lié aux maladies chroniques de ces régions est le plus important au Canada ${ }^{39,40}$. La population y rencontre également de nombreux obstacles à l'accès aux soins. Le sous-développement de l'auto-prise en charge pourrait s'expliquer par l'existence de priorités concurrentes en matière de santé et par la dispersion de la population sur le territoire.

Il existe un grand potentiel d'amélioration de la santé dans le Nord, étant donné que les programmes d'auto-prise en charge des maladies chroniques les plus courants et les plus efficaces ${ }^{15,41}$ sont fondés sur un modèle de soutien par les pairs qui ne dépend pas de l'accès à des professionnels de la santé formés. En outre, bon nombre de programmes ont déjà été adaptés à de nombreuses cultures et dans diverses langues et ont été mis en œuvre avec succès ${ }^{42-44}$.

Le Canada dispose de nombreuses stratégies axées sur une maladie dont l'un des thèmes est l'auto-prise en charge. Par exemple, les programmes de soutien à l'auto-prise en charge en Ontario sont principalement financés dans le cadre de la Stratégie ontarienne de lutte contre le diabète. La capacité d'intégrer les soins sur le plan programmatique est donc réduite, étant donné que les mesures du rendement sont alors souvent liées à une maladie plutôt qu'à une population. Même si les soins liés au diabète sont souvent considérés comme une première étape ou un modèle permettant d'aborder les mala- 
dies chroniques, les approches d'autoprise en charge du diabète demeurent étroitement associées à la prise en charge médicale propre à la maladie, notamment les connaissances sur le diabète et l'apprentissage des tâches médicales (p. ex. l'utilisation de l'insuline). De plus, comme les diabétiques forment la population visée par ce programme de soutien à l'auto-prise en charge, cela a généralement pour effet d'exclure les personnes atteintes d'autres maladies chroniques.

Il est essentiel de maintenir l'accent sur une approche plus générale (gestion de la fatigue, plan d'action pour un style de vie sain, etc.) qui aborde les trois dimensions de l'auto-prise en charge: la prise en charge médicale de la maladie par les patients, l'exercice des activités et des rôles habituels et la maîtrise des répercussions émotionnelles ${ }^{15}$. L'accent sur les facteurs de risque communs à l'ensemble des maladies chroniques est un principe fondamental de l'approche du modèle de soins aux malades chroniques ${ }^{36}$. Selon les recommandations de l'Organisation mondiale de la Santé, " une politique gouvernementale saine et explicite est indispensable pour assurer l'efficacité des mesures de prévention et de lutte contre les maladies chroniques. ${ }^{45}, \mathrm{p} .2$. Une stratégie générale qui adopte une perspective axée sur le parcours de vie et qui est coordonnée par les décideurs de l'ensemble des secteurs est recommandée ${ }^{45}$.

L'Alberta et la Colombie-Britannique, les provinces dont les approches d'auto-prise en charge semblent les plus complètes, possèdent aussi les politiques et les stratégies les plus détaillées qui ne sont pas axées sur une maladie en particulier. Les soins de santé primaires et les soins primaires y occupent une place centrale. Le rôle du fournisseur de soins primaires peut être considéré comme essentiel au soutien de l'auto-prise en charge par le patient. La nature des soins primaires et la position qu'ils occupent au sein du système de santé en font une cible parfaite pour de telles interventions. Le secteur des soins primaires a non seulement accès à la plupart des patients atteints d'une maladie chronique, mais il s'attaque également à divers problèmes de santé et non une seule maladie. Les fournisseurs de soins primaires sont dans une position idéale pour jouer un rôle central dans la prévention et la prise en charge des maladies chroniques, étant donné que $95 \%$ des Canadiens atteints d'une maladie chronique déclarent avoir un médecin de famille $^{46}$. Les consultations en soins primaires offrent une occasion unique de surveiller la santé des patients et d'encourager l'auto-prise en charge ${ }^{47-49}$, puisque la majorité des Canadiens perçoivent leur médecin de famille comme une source crédible de renseignements sur la santé et respectent ses conseils ${ }^{50,51}$. Comme ces provinces vont de l'avant avec des stratégies qui sont davantage ancrées dans le milieu des soins de santé primaires que dans les secteurs axés sur une maladie, il est important d'évaluer les répercussions des diverses politiques provinciales sur la portée du programme et son efficacité en général. Jusqu'à maintenant, peu de données publiées décrivent la portée globale des programmes de soutien à l'auto-prise en charge dans l'ensemble des provinces ${ }^{52}$.

Il y a lieu de mener d'autres recherches visant à évaluer l'association entre la portée des politiques et des programmes et l'effet sur l'auto-prise en charge des maladies chroniques.

\section{Limites}

Les résultats de cette étude sont limités par plusieurs facteurs, notamment un biais de participation et des questions liées au calendrier de l'étude. Nous nous sommes principalement fiés à la première liste de représentants des provinces et des territoires fournie par le CCS. Même si nous avons discuté avec des représentants de l'ensemble des provinces et des territoires et que nous avons assuré un suivi à des fins de vérification ou de clarification au besoin, le degré de connaissances des divers représentants variait, probablement en fonction de la durée de leur présence en poste et de leur connaissance générale du système gouvernemental. Ces aspects n’ont pas été expressément évalués.

De plus, une limite courante des analyses de politiques est lié au fait qu'une bonne partie du matériel a une durée de vie limitée et est lié aux programmes politiques et aux déclarations publiques. Ce matériel n’était donc pas nécessairement accessible au public au moment où nous avons effectué la recherche. Nous avons tenté d'atténuer cette limite en interviewant des experts du domaine ainsi qu'en effectuant une nouvelle analyse en ligne à la suite des entrevues, en juillet 2012 .

\section{Conclusion}

Les données laissent penser que la participation des patients à l'auto-prise en charge de leur maladie chronique est importante et efficace. Même si la plupart des provinces et des territoires ont des politiques qui englobent des éléments de l'auto-prise en charge des maladies chroniques, ces politiques font souvent partie d'autres initiatives ou relèvent de documents de politiques axés sur des maladies ou des populations en particulier. Cette approche pourrait limiter la portée et l'effet potentiel de l'auto-prise en charge. L'élaboration de politiques dans lesquelles l'auto-prise en charge serait un élément clé d'une approche visant l'ensemble de la population pourrait améliorer les soins aux Canadiens atteints de maladies chroniques.

\section{Remerciements}

Ce rapport a été financé par le Conseil canadien de la santé dans le cadre de la rédaction d'un document technique portant sur l'auto-prise en charge des maladies chroniques. Les auteurs aimeraient remercier Mary Byrnes du Conseil canadien de la santé, qui a assuré la liaison avec les représentants des provinces et des territoires pour les entrevues, ainsi que la réviseure médicale en poste à Ottawa Joan Ramsay, qui a aidé à la rédaction de cet article.

\section{Références}

1. Secrétariat du Réseau intersectoriel de promotion des modes de vie sains; Groupe de travail F-P-T sur les modes de vie sains; Comité consultatif F-P-T sur la santé de la population et la sécurité de la santé (CCSPSS). La Stratégie pancanadienne intégrée en matière de modes de vie sains. Ottawa (Ont.): ministère de la Santé; 2005. PDF $(232,44 \mathrm{Ko})$ téléchargeable à partir du lien: http://www.phac-aspc .gc.ca/hl-vs-strat/pdf/hls_f.pdf 
2. Alliance pour la prévention des maladies chroniques au Canada. Prévention intégrée des maladies chroniques - Ensemble en force : Un appel à l'action. Deuxième conférence nationale de l'Alliance pour la prévention des maladies chroniques au Canada (APMCC), novembre 2006. Ottawa (Ont.) : Alliance pour la prévention des maladies chroniques au Canada; 2007. PDF (26 Ko) téléchargeable à partir du lien: http://www.cdpac.ca/media.php?mid = 309

3. Fortin $\mathrm{M}$, Bravo $\mathrm{G}$, Hudon $\mathrm{C}$ et collab. Relationship between multimorbidity and health-related quality of life of patients in primary care. Qual Life Res. 2006;15(1): 83-91.

4. Fortin M, Bravo G, Hudon C, Lapointe L, Dubois MF, Almirall J. Psychological distress and multimorbidity in primary care. Ann Fam Med. 2006;4(5):417-22.

5. Fortin M, Soubhi H, Hudon C, Bayliss EA, van den Akker M. Multimorbidity's many challenges. BMJ. 2007;334(7602):1016-7.

6. Hansagi H, Olsson M, Sjoberg S, Tomson Y, Goransson S. Frequent use of the hospital emergency department is indicative of high use of other health care services. Ann Emerg Med. 2001;37(6):561-7.

7. Incalzi RA, Capparella O, Gemma A et collab. The interaction between age and comorbidity contributes to predicting the mortality of geriatric patients in the acutecare hospital. J Intern Med. 1997;242(4): 291-8.

8. Librero J, Peiro S, Ordinana R. Chronic comorbidity and outcomes of hospital care: length of stay, mortality, and readmission at 30 and 365 Days. J Clin Epidemiol. 1999;52(3):171-9.

9. Poses RM, McClish DK, Smith WR, Bekes C, Scott WE. Prediction of survival of critically ill patients by admission comorbidity. J Clin Epidemiol. 1996;49(7):743-7.

10. Rochon PA, Katz JN, Morrow LA et collab. Comorbid illness is associated with survival and length of hospital stay in patients with chronic disability. A prospective comparison of three comorbidity indices. Med Care. 1996;34(11):1093-101.
11. Committee on Quality of Health Care in America. Crossing the quality chasm: a new health system for the 21st century. Washington (DC) : National Academies Press; 2001.

12. Anderson RM, Funnell MM, Butler PM, Arnold MS, Fitzgerald JT, Feste CC. Patient empowerment. Results of a randomized controlled trial. Diabetes Care. 1995;18(7): 943-9.

13. Vickery DM, Kalmer H, Lowry D, Constantine M, Wright E, Loren W. Effect of a self-care education program on medical visits. JAMA. 1983;250(21):2952-6.

14. Bodenheimer $\mathrm{T}$, Lorig $\mathrm{K}$, Holman $\mathrm{H}$, Grumbach K. Patient self-management of chronic disease in primary care. JAMA. 2002;288(19):2469-75.

15. Lorig KR, Holman HR. Self-management education: History, definitions, outcomes, and mechanisms. Annals of Behavioral Medicine. 2003;26(1):1.

16. McGowan P. The Chronic Disease SelfManagement Program in British Columbia. In: Dorland J, McColl MA, editors. Emerging approaches to chronic disease management in primary health care: managing chronic disease in the twenty-first century. Kingston (Ont.) : Queen's University School of Policy; 2007. p. 79-90.

17. Newman S, Steed E, Mulligan K. Chronic physical illness: self-management and behavioural interventions. Maidenhead (UK) : Open University Press; 2009.

18. Pearson ML, Mattke S, Shaw R, Ridgely S, Wiseman SH. Patient self-management support programs: an evaluation [Internet]. Agency for Healthcare Research and Quality, US Department of Health and Human Services; 2007 [consultation le 5 novembre 2012]. Consultable en ligne à la page: http://www.ahrq.gov/qual/ptmgmt /index.html\#contents

19. Wagner EH, Austin BT, Von Korff M. Organizing care for patients with chronic illness. Milbank Q. 1996;74(4):511-44.

20. Zwar N, Harris M, Griffiths R et collab. A systematic review of chronic disease management. Canberra (AU) : Australian Primary Health Care Research Institute; 2006.
21. Adams K, Greiner AC, Corrigan JM, editors. Report of a summit. The 1st annual crossing the quality chasm summit - A focus on communities. Washington (DC) : National Academies Press; 2004.

22. Johnston SE, Liddy CE, Ives SM. Selfmanagement support: a new approach still anchored in an old model of health care. Can J Public Health. 2011;102(1):68-72.

23. Edwards P, Mawani A; Le Alder Group pour le Groupe de travail sur le vieillissement en santé et le mieux-être du Comité fédéral-provincial-territorial des hauts fonctionnaires responsables des aînés. Le vieillissement en santé au Canada : une nouvelle vision, un investissement vital Document de discussion 3, Les moyens de concrétiser la vision [Internet]. Ottawa (Ont.) : Agence de la santé publique du Canada; [2013]. Consultable en ligne à la page : http://www.phac-aspc.gc.ca/seniors -aines/publications/public/healthy-sante /vision/vision-bref/chap03-fra.php

24. Agence de la santé publique du Canada. La Stratégie canadienne du diabète : historique, évolution et avenir [Internet]. Ottawa (Ont.) : Agence de la santé publique du Canada; [modifié le 18 février 2013; consultation le 23 avril 2013]. Consultable en ligne à la page : http://www.phac-aspc.gc.ca/cd-mc /diabetes-diabete/strategy_funding-strategie finance-fra.php

25. Conseil canadien de la santé. Soutien à l'autogestion pour les Canadiens atteints de maladies chroniques : point de mire sur les soins de santé primaire. Toronto (Ont.) : Conseil canadien de la santé; 2012.

26. Liddy CE, Johnston S, Mill K, Shahidi J. Self-management support interventions: technical paper on the sustainability of self-management supports for people with chronic conditions and the availability of self-management support programs for people with multiple chronic conditions. Document non publié; [2011].

27. Broemeling AM, Watson DE, Prebtani F. Population patterns of chronic health conditions, co-morbidity and healthcare use in Canada: implications for policy and practice. Healthc Q. 2008;11(3):70-6.

28. Buse K, Mays N, Walt G. Making health policy. Maidenhead (UK) : Open University Press; 2005. 
29. Partenariat canadien contre le cancer. Analyse environnementale des politiques et de la législation en matière de prévention du cancer de la peau : sommaire. Winnipeg (Man.) : Groupe d'action pour la prévention primaire; 2009.

30. Bergeron K. Ontario Heart Health Network: collaborative policy scan project - final report. Toronto (Ont.): Ontario Heart Health Network; 2010. PDF (4,81 Mo) téléchargeable à partir du lien: http://www .goforhealth.ca/Areas/Custom/ContentFiles /GFH\%20Resources/Full_OHHN_PolicyScan \%20march_2010.pdf

31. McGrath C, Myers J. Policies that Support Bridging, Bonding and Building Between Government and the Social Economy in Atlantic Canada: policy scan process report 2009. Halifax (N.-É.) : Social Economy and Sustainability Research Network; 2009.

32. Dixon-Woods M, Agarwal S, Jones D, Young B, Sutton A. Synthesising qualitative and quantitative evidence: a review of possible methods. J Health Serv Res Policy. 2005 Jan;10(1):45-53.

33. Alberta Health and Wellness. Vision 2020: The Future of Health Care in Alberta. Edmonton (AB) : Alberta Health and Wellness; 2008.

34. Government of British Columbia. Primary Health Care Charter: a collaborative approach. Victoria (BC) : British Columbia Ministry of Health;[2012]. PDF (708,49 Ko) téléchargeable à partir du lien : http://www .health.gov.bc.ca/library/publications/year /2007/phc_charter.pdf

35. Barr VJ, Robinson S, Marin-Link B et collab. The expanded Chronic Care Model: an integration of concepts and strategies from population health promotion and the Chronic Care Model. Hosp Q. 2003;7(1): 73-82.

36. Wagner EH, Austin BT, Davis C, Hindmarsh M, Schaefer J, Bonomi A. Improving chronic illness care: translating evidence into action. Health Affairs. 2001;20(6):64-78.

37. Lemmens KM, Nieboer AP, van Schayck CP, Asin JD, Huijsman R. A model to evaluate quality and effectiveness of disease management. Qual Saf Health Care. 2008 Dec;17(6):447-53.
38. Improving health together: a policy framework for chronic disease prevention and management in Newfoundland and Labrador. St. John's (NL) : Newfoundland and Labrador Department of Health and Community Services; [2011]. PDF (9,14 Mo) téléchargeable à partir du lien: http://www .health.gov.nl.ca/health/chronicdisease /Improving_Health_Together.pdf

39. Bruce SG, Riediger ND, Zacharias JM, Young TK. Obésité et affections liées à l'obésité dans une population des Premières nations du Canada. Maladies chroniques et blessures au Canada. 2010; 31(1):32-8.

40. Initiative sur le diabète chez les Autochtones. Le diabète dans les populations autochtones (Premières nations, Inuits et Métis) du Canada : les faits. Ottawa (Ont.) : Santé Canada; 2001.

41. Brady TJ, Murphy L, Beauchesne D et collab. Sorting through the evidence for the Arthritis Self-Management Program and the Chronic Disease Self-Management Program: executive summary of ASMP/ CDSMP meta-analyses. Atlanta (GA) : Centers for Disease Control and Prevention; 2011. PDF (1,20 Mo) téléchargeable à partir du lien : www.cdc.gov /arthritis/docs/ASMP-executive-summary .pdf

42. Barceló A, Cafiero E, de Boer M et collab. Veracruz Project for the improvement of diabetes care (VIDA) - final report. Washington (DC) : Pan American Health Organization; 2010.

43. Griffiths C, Motlib J, Azad A et collab. Randomised controlled trial of a lay-led selfmanagement programme for Bangladeshi patients with chronic disease. Br J Gen Pract. 2005;55(520):831-7.

44. Fu D, Fu H, McGowan $\mathrm{P}$ et collab. Implementation and quantitative evaluation of chronic disease self-management programme in Shanghai, China: randomized controlled trial. Bull World Health Organ. 2003;81(3):174-82.
45. Organisation mondiale de la Santé. Note d'orientation - Prévention des maladies chroniques : conception et mise en œuvre de politiques efficaces. Genève $(\mathrm{CH})$ : Organisation mondiale de la Santé; [s. d., consulté le 30 mai 2013]. PDF (473,72 Ko) téléchargeable à partir du lien : http://www .who.int/chp/advocacy/policy.brief_FR_web .pdf

46. Glazier RH, Moineddin R, Agha M et collab. The impact of not having a primary care physician among people with chronic conditions. ICES Investigative Report Toronto (Ont.) : Institute for Clinical Evaluative Sciences; 2008.

47. Chan BT. The declining comprehensiveness of primary care. CMAJ. 2002;166(4):429-34.

48. Cifuentes M, Fernald DH, Green LA et collab. Prescription for health: changing primary care practice to foster healthy behaviors. Ann Fam Med. 2005;3 Suppl 2: S4-11.

49. Hensrud DD. Clinical preventive medicine in primary care: background and practice: Rationale and current preventive practices. Mayo Clin Proc. 2000;75(2):165-72.

50. Folsom AR, Grimm RH Jr. Stop smoking advice by physicians: a feasible approach? Am J Public Health. 1987;77(7):849-50.

51. Green LA, Fryer GE Jr., Yawn BP, Lanier D, Dovey SM. The ecology of medical care revisited. N Engl J Med. 2001;344(26): 2021-5.

52. Johnston S, Liddy $\mathrm{C}$, Mill $\mathrm{K}$, Irving $\mathrm{H}$. Building the evidence base for chronic disease self-management support interventions across Canada. Can J Public Health. 2012;103(6):e462-67. 